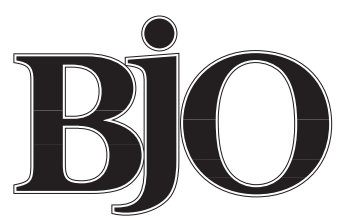

British Journal of Ophthalmology

\title{
Editorials
}

\section{New series on management controversies in ophthalmology}

Ophthalmology has become divided into a number of subspecialties and in each considerable experience and expertise have developed. Clinically, this expertise is a tremendous asset when a unit is large enough to have most of the subspecialties represented. However, many smaller units do not have this advantage and yet still have to manage similar problems, albeit less frequently.

In this new series, we have invited experts in each major subspecialty to examine a clinical problem within their subspecialty in which there are a variety of views or management options. Several formats for these clinical controversies have arisen and our guest experts have chosen at least two collaborators, often from different continents, who usually have differing views and experience to contribute. These different perspectives are discussed and our invited expert then draws the views together and provides a rational approach to the problem.

Our aim is to cover most of the subspecialties and discuss problems within them that are relevant to the general ophthalmologist. We hope that this series will be of interest to you and useful in your clinical practice.

We start the series in this issue with David Taylor as our guest expert and he looks at the management of congenital cataracts.

SUSAN LIGHTMAN

Moorfields Eye Hospital, London EC1V 2PD, UK

PETER MCCLUSKEY

St Vincent's Hospital and Royal Prince Alfred Hospital, Sydney, Australia

\section{Retinopathy of prematurity—the "second lull"?}

Ophthalmologists who trained in the 1960s and 1970s believed that retrolental fibroplasia was a tragic, iatrogenic problem of the 1940s and early 1950s. In the 1960s and 1970 s screening programmes were generally not in place, as this was the period of the "first lull"- the first epidemic (due to unmonitored supplemental oxygen, often given routinely to all premature babies) ${ }^{1}$ was over, and the "second epidemic" (due to increased survival of very low birth weight babies) had not really started. The paper by Rowlands et al suggests that we may be entering a "second lull." 2 The study reports the changing incidence of retinopathy of prematurity (ROP) in babies in one unit in the United Kingdom over a 10 year period (1989-98), and shows a significantly lower incidence of stage 3-5 ROP in babies examined in the second 5 year period compared with the first. This is despite babies in the second cohort being more preterm with lower birth weights as a result of improved survival of extremely low birthweight babies. If the risk factors for ROP had remained constant over the study period, one would expect a higher incidence in the second cohort, given that birth weight and gestational age are the most important risk factors. The authors conclude that the lower incidence of ROP in babies born more recently is attributable to improvements in neonatal care, in particular to the use of prenatal steroids and surfactant. However, this statement has to be interpreted cautiously, as historical cohort studies are subject to inherent weaknesses.

This paper raises several questions concerning the incidence of severe ROP. Firstly, has a fall in the incidence of ROP been reported in other studies, and if so, is this a universal trend? Secondly, if the trend is real, what are possible explanations? Thirdly, what are the implications for clinical practice and research, and lastly, are these applicable universally?

Rowlands quotes studies from the USA, Denmark, and the UK which also report a reduction in the incidence of ROP over time, or where the incidence of ROP has remained stable despite more extremely premature babies being included. These studies seem to have provoked considerable controversy not only regarding whether the trend is real, but also over possible explanations. Comparison between neonatal units has to be interpreted with caution, as differences in the population of babies referred to the units may, in part, explain differences in incidence. However, there is an increasing body of evidence that suggests that the incidence and severity of ROP in babies $<1250 \mathrm{~g}$ at birth is decreasing in industrialised countries. 
One has to be aware that this trend may not be universaldata from Latin American countries, and urban centres in developing countries such as India, show that babies with a much wider range of birth weights are developing threshold disease than in industrialised countries. ${ }^{34}$ The evidence suggests that in settings where neonatal care is inadequate, risk factors relevant during the "first epidemic" (that is, unmonitored supplemental oxygen) lead to severe ROP in more mature babies and the more premature babies are dying. Conversely, more sophisticated units reflect the "second epidemic," with more premature babies developing ROP. ${ }^{5}$

In the paper by Rowlands and colleagues the authors suggest that improved neonatal care explains the falling incidence in birthweight specific cohorts of babies, but how good is the evidence for this? Clinical trials of interventions in preterm babies are complicated by the fact that the organs of very premature babies are still differentiating as well as growing - the time of onset of the interventions as well as the dose, duration, and cessation of the intervention (for example, abrupt versus gradual), may all be relevant variables. Some trials have been designed to determine whether the intervention will prevent the outcome of interest, or to assess effectiveness once the condition has developed.

A review of the Cochrane Collaboration Neonatal Group's list of systematic reviews shows that 106 reviews of clinical trials have been undertaken, of which 74 are for interventions targeted at preterm babies (website: http:/www.update-software.com/ccweb/cochrane/revabstr/ g030index.htm). The studies have reviewed clinical trials undertaken to investigate a wide range of interventions to improve neonatal outcomes, including control of insensible fluid loss; different methods of ventilation; different regimes for supplemental feeding; the role of synthetic or natural surfactants for the prevention or treatment of respiratory distress syndrome (RDS); dopamine and albumin treatment for hypotension; the timing, dose, and method of administration of steroids to prevent or treat chronic lung disease; the timing and speed of discontinuation of oxygen, and interventions to control body temperature. The wide range and quality of the trials, as well as the large number of systematic reviews, shows a highly commendable emphasis on evidence based medicine.

Many of the studies undertaken did not include ROP as an main, or subsidiary, outcome but several did report the incidence of ROP. These studies can be divided into two groups: those which might have an impact on the development of ROP through stabilising the respiratory system and/or the newborn's physiology, and those which could arguably have a direct bearing on mechanisms implicated in the pathogenesis of ROP (that is, oxygen supplementation regimes, light exposure, antioxidant treatment). In the first group the following interventions were associated with a significantly lower risk of ROP: D-penicillamine treatment to prevent hyperbilirubinaemia, and treatment of infants with RDS with inositol (which promotes maturation of surfactant). Studies that did not show a statistically significant reduction in the incidence of ROP (usually severe ROP) include postnatal steroids given within 96 hours of birth, natural or synthetic surfactant given prophylactically to prevent RDS, or given as a treatment for established RDS. An insignificant increase in ROP was observed in studies where steroids were given more than 3 weeks after birth.

In the second group of trials the incidence of ROP was less in babies in whom discontinuation of oxygen was gradual rather than abrupt, and where the use of oxygen was restricted. Early versus late discontinuation of oxygen did not have any impact on the development of ROP. A systematic review of studies restricting ambient light has also failed to demonstrate a reduction in ROP. A vitamin A supplementation trial showed a non-significant trend for a lower incidence of ROP in the supplemented group. Rather surprisingly, the Cochrane Neonatal Group has not undertaken a systematic review of the role of vitamin $\mathrm{E}$ in preventing ROP, where at least six clinical trials have been undertaken. A meta-analysis of these studies ${ }^{6}$ has not helped to resolve the current controversy, but it has been argued that an adequately powered clinical trial is justified, requiring a sample size of 2600 babies. $^{7}$

There are other, important clinical trials which are not included in the Cochrane Library. One is the STOP-ROP trial, which was designed to determine whether higher oxygen saturation once prethreshold disease had developed would prevent progression to threshold disease requiring treatment. ${ }^{8}$ Babies with prethreshold disease were randomly allocated to two groups: one group received supplemental oxygen so that oxygen saturation was maintained at $96-99 \%$ (supplemented group), while in the second group it was maintained at $89-94 \%$ (conventional group). The study showed that the rate of progression in the supplemental group was lower than in the conventional group, but the findings were not statistically significant. A subgroup of babies without "plus" disease at enrolment seemed to be more responsive to supplemental oxygen. Other, retrospective studies of oxygen supplementation have recently been reported, which showed better results. However, these studies were not randomised clinical trials and the findings need to be interpreted cautiously. The evidence that antenatal dexamethasone reduces the incidence and severity of ROP is also not derived from clinical trials.

The rate of adverse events is another important aspect to consider in trials. In the STOP-ROP trial the rate of chronic lung disease was higher in the "supplemental" group than in the conventional group, and in the vitamin $\mathrm{E}$ trials there was a higher rate of a variety of adverse events (but not mortality) in supplemented babies. The potential for harm has to be balanced against potential for benefit, with implications not only for clinical practice, but also as further clinical trials may not be considered ethical.

To summarise, many trials to assess interventions to improve neonatal outcomes have been undertaken, and some clearly show an impact on the development of ROP.

What are the clinical implications of a falling incidence of severe ROP? Several authors have critically reviewed the criteria used for screening, which in the UK are a birth weight of $\leqslant 1500 \mathrm{~g}$, or a gestational age of $<32$ weeks (in the USA the criteria are a birth weight $\leqslant 1500$ g; gestational age $<29$ weeks, or babies weighing $>1500 \mathrm{~g}$ with an "unstable clinical course"). As the vast majority of babies who develop threshold disease fall within these criteria, reducing the birthweight criterion to $\leqslant 1250 \mathrm{~g}$ has been suggested. ${ }^{9}$ However, lowering the birthweight criterion would expose a small number of larger babies who are at risk, and research is needed to clearly establish relevant "sickness criteria" in babies weighing $>1250 \mathrm{~g} .{ }^{10}$ In an ideal world an objective test would be the best way of identifying babies at risk of severe ROP, and a recent study suggests that serum vascular endothelial growth factor (VEGF) may prove to be a useful predictive tool. ${ }^{11}$ In middle income countries and in urban settings in developing countries, screening criteria may need to vary widely depending on the level of care being provided, and some 
centres may need to include babies weighing $\leqslant 1750 \mathrm{~g}$ at birth. $^{3-5}$ Research into this important area is urgently needed so that the tragedy of the "first epidemic" can be avoided in countries which are expanding neonatal intensive care services.

There are still many unknowns concerning the factors that initiate and promote the progression of ROP. ${ }^{12}$ Oxygen almost certainly plays an important part in both processes, and further research is warranted into optimum oxygen saturation levels, which may vary depending on postconceptual age. Other important areas of research include early nutritional interventions, exploration of objective tests which have a high positive predictive value for the development of severe ROP, and examination of the role of non-ophthalmologists for preliminary screening for $\mathrm{ROP}^{13}$ in settings where ophthalmologists skilled at indirect ophthalmoscopy are in short supply.

CLARE GILBERT

Department of Epidemiology and International Eye Health, Institute of Ophthalmology, 11-43 Bath Street, London EC1V 9EL, UK clare.gilbert@ucl.ac.uk
1 Jacobson RM, Feinstein AR. Oxygen as a cause of blindness in premature infants: "autopsy" of a decade of errors in clinical epidemiological research. f Clin Epidemiol 1992;45:1265-87.

2 Rowlands E, Ionides ACW, Chinn S, et al. Reduced incidence of retinopathy of prematurity. Br f Ophthalmol 2001;85:933-5.

3 Report of workshop on retinopathy of prematurity for ophthalmologists and neonatologists from six Latin American countries, Quito, Chile. London: Department of Epidemiology and International Eye Health, 1997.

4 Charan R, Dogra MR, Gupta A, et al. The incidence of retinopathy of prematurity in a neonatal care unit. Indian f Ophthalmol 1995;43:123-6.

5 Gilbert C, Rahi J, Eckstein M, et al. Retinopathy of prematurity in middleincome countries. Lancet 1997;350:12-14.

6 Raju TNK, Langenberg P, Bhutani V, et al. Vitamin E prophylaxis to reduce retinopathy of prematurity: a reappraisal of published trials. Paediatrics 1997;131:844-50.

7 Zamora SA, Maret A. Vitamin E for ROP. F Paediatr 1999;134:249.

8 The STOP-ROP Multicentre Study Group. Supplemental therapeutic oxygen for prethreshold retinopathy of prematurity (STOP-ROP), a randomised, controlled trial, I: Primary outcomes. Paediatrics 2000;105: randomised

9 Goble RR, Jones HS, Fielder AR. Are we screening too many babies for retinopathy of prematurity? Eye 1997;11:509-14.

10 Pollock W, Inglesby DV, Cottrell DG. Letter. Eye 1998;12:1035.

11 Brady-McCreery KM, McCreery CJ, Contant C, et al. Serum vascular endothelial growth factor and retinopathy of prematurity. Invest Ophthalmol Vis Sci (Suppl) 2001;42:S682.

12 Fielder AR. Retinopathy of prematurity: aetiology. Clinical Risk 1997;3:4751.

13 Saunders RA, Bluestein EC, Berland JE, et al. Can non-ophthalmologists screen for retinopathy of prematurity? 7 Pediatr Ophthalmol Strabismus 1995;32:302-4.

\section{Who needs an iridotomy?}

Angle closure glaucoma (ACG) is one of the leading causes of global blindness. Recent population based research on Chinese subjects in Singapore and a southern Indian population found high rates of ACG among those populations. ${ }^{12}$ Close to $2 \%$ of individuals over the age of 40 were found to have ACG in these studies. Given that almost half of the world's population lives in China and India, millions of individuals are at risk of ACG and may benefit from better screening strategies to identify them before glaucoma develops. ${ }^{3}$

However, the decision to perform a laser peripheral iridotomy (LPI) on a patient with a narrow angle is often highly subjective. What is an "occludable" angle? If one can see trabecular meshwork is the patient "safe." If one cannot, is the patient at significant risk? What proportion of the angle needs to be visible? What should be done in the developing world setting where an ACG suspect is unlikely to receive a second eye examination in the near future?

A recent World Health Organization (WHO) sponsored meeting on glaucoma blindness worldwide proposed that a new nomenclature be employed for describing individuals with ACG. Those with 90 degrees of angle in which the trabecular meshwork is visible who have glaucomatous optic nerve damage have "primary ACG," while those with similarly closed angles and elevated eye pressure or peripheral anterior synechiae (PAS) have "primary angle closure." The term glaucoma is only used for those with glaucomatous optic neuropathy and visual field loss. A third, important category of patients is those with narrow angles as described above with no evidence of glaucoma or damage to the angle (that is, no elevated IOP or peripheral anterior synechiae). These individuals are simply described as having "narrow angles," not having angle closure glaucoma. How to manage this large group of asymptomatic individuals is not well documented in the literature and poses a major public health problem if screening programmes are to be undertaken in the developing world.
Another source of confusion when discussing ACG is the co-mingling of acute and chronic angle closure. Acute ACG has classic signs and symptoms and is typically relieved by laser iridotomy. Several studies have found from $15 \%$ to $45 \%$ of all ACG is acute. ${ }^{1-5}$ The majority of cases are therefore chronic ACG, which may behave quite differently from acute ACG. It is not clear if laser iridotomy is effective at stopping the progression of chronic ACG once it starts.

Prophylactic laser iridotomy has been proved to be beneficial for individuals who have suffered a monocular attack of acute ACG. Lowe documented in the 1950s that when the contralateral eye of individuals suffering acute ACG was treated either with nothing or with pilocarpine once or twice a day, $50 \%$ developed acute attacks over a period of up to 25 years. ${ }^{6}$ Conversely, only one of 54 individuals treated with prophylactic surgical iridectomy during this same time developed an acute attack. Edwards reported a high likelihood of developing an acute attack in the untreated fellow eye in the first month. ${ }^{7}$ Snow also documented high rates of attacks in untreated contralateral eyes. ${ }^{8}$ Clearly, contralateral eyes of patients with acute ACG are at significant risk for an acute attack and iridectomy virtually eliminates this risk.

The study by Lowe described above reported the effectiveness of surgical iridectomy in preventing acute attacks in second eyes. Fleck et al randomised individuals to surgical or laser iridectomy and obtained similar results. ${ }^{9}$ A recent retrospective study of patients undergoing laser peripheral iridotomy in the contralateral eye in Singapore also found excellent results with this treatment. ${ }^{10}$

The literature is less clear about the natural history of untreated individuals who have gonioscopically narrow angles. Wilensky and colleagues enrolled 129 mostly European derived subjects with "occludable" angles and central anterior chamber depth (measured by optical pachymetry) less than $2.0 \mathrm{~mm}$ in a prospective study. ${ }^{11}$ Patients were recruited over a 5 year period at five separate centres. Eight patients $(6.2 \%)$ developed acute angle closure glaucoma (AACG) and $17(13.2 \%)$ developed either 
appositional closure or peripheral anterior synechiae in at least 0.5 clock hours of the superior quadrant (median follow up 3 years). Dark room prone provocative testing did not consistently predict who would develop angle closure during follow up. This study in European derived individuals clearly demonstrates that a combined screening strategy using anterior chamber depth and gonioscopy had a low positive predictive value for the development of acute attacks.

Alsbirk examined 75 Greenland Eskimos with shallow central anterior chamber depth and a van Herick score of two or less 10 years after a baseline examination. ${ }^{12} \mathrm{He}$ had performed gonioscopy on 69 of these individuals at the baseline. Of the 20 individuals felt to have "occludable" angles at baseline, seven (35\%) developed ACG, as opposed to four of 49 (8\%) felt to be non-occludable. However, of these 11 cases, two were acute attacks and only one other had peripheral anterior synechiae associated with elevated eye pressure. The remainder had either intermittent symptoms or synechiae on gonioscopy. Furthermore, this population has a very high risk of ACG, with $1 \%$ of individuals over the age of 60 blind from this disease.

These two articles sum up the English literature on how well a physician seeing a gonioscopically narrow angle predicts the later development of ACG in untreated eyes. Only European derived individuals being followed by glaucoma specialists and Greenland Eskimos being examined by a single highly trained observer were studied. No data are published in the English literature on Asian populations followed longitudinally. How should clinicians and public health officials behave when identifying individuals with narrow angles who have limited access to healthcare services given the paucity of data?

Theoretically, laser peripheral iridotomy should prevent the onset of chronic ACG as well as acute ACG, although once chronic ACG is established, limited evidence suggests that laser peripheral iridotomy may be insufficient to control intraocular pressure. ${ }^{13}{ }^{14}$ Chronic ACG may be associated with higher rates of blindness than is typically found with open angle glaucoma. ${ }^{1}$ This has led some to advocate more aggressive screening so that early laser peripheral iridotomy can be performed in individuals with "high risk" of either acute ACG or chronic ACG.

While laser peripheral iridotomy appears relatively harmless, one cannot be certain of the long term safety of this procedure. laser peripheral iridotomy disrupts the natural flow of aqueous in the eye and results in significant increase in lens-iris contact. ${ }^{15}$ Theoretically, this may predispose to a more rapid development of cataract since less aqueous is in contact with the lens epithelium. Several studies have attempted to look at this issue, but follow up has been short, no lens grading system was used, and no acceptable control groups were studied. ${ }^{16}{ }^{17}$ Focal lenticular opacities seen after argon laser peripheral iridotomy are said not to progress, but once again, follow up has been short in published reports. The plausibility of laser peripheral iridotomy being able to cause cataract is supported by the strong evidence in the literature that trabeculectomy can do just this in glaucoma patients. ${ }^{18}$

Laser energy delivered at the time of treatment as well as the altered fluidics of the eye may have other ramifications including the hastening of corneal endothelial cell dysfunction. Once again, researchers looking at specular microscopy have only studied small numbers of individuals at relatively short follow up. ${ }^{16}{ }^{19}$ Argon laser peripheral iridotomy has been consistently reported to cause localised transient corneal oedema, ${ }^{17}{ }^{20}$ with rare case reports of corneal decompensation. ${ }^{21}$ One study documented a higher rate of endothelial cell loss after argon laser peripheral iridotomy than after YAG laser peripheral iridotomy. ${ }^{16}$ Another potential complication of laser peripheral iridotomy is the development of posterior synechiae following laser iridotomy. ${ }^{17}$ Posterior synechiae can both limit vision in dim environments and make later cataract surgery more challenging.

While these complications seem minor when compared with the risk of an acute attack, they are in fact of significant concern for individuals contemplating glaucoma control programmes in developing countries with high prevalence of narrow angles. Visually significant complications that affect as few as $5 \%$ of those treated can be devastating to such programmes. If laser peripheral iridotomy hastens cataract significantly, one could cause more blindness with widespread screening and iridotomy treatment than one prevents in developing countries where cataract services are not universally available.

Fifteen per cent of Mongolian women over 50 years of age have occludable angles as defined by an observer being unable to see pigmented trabecular meshwork for more than 90 degrees on gonioscopy with a Goldmann lens. A similar proportion of Chinese residents of Singapore were found to have occludable angles in a recently completed population based survey. Assuming similar rates among older women in China, then about 25 million Chinese women and 12 million men will be eligible for prophylactic laser peripheral iridotomy in the year 2010. The numbers may be even higher in India. A complication that occurs in only $5 \%$ of those treated will adversely impact close to two million individuals assuming that all are identified and treated.

Before embarking on large scale screening campaigns and investing resources in expanding prophylactic treatment of these individuals, data need to be collected on the natural history of treated and untreated narrow angles. Research should be conducted to identify factors at baseline that predict who, with narrow angles, is at greatest risk of developing either acute or chronic angle closure glaucoma and, ultimately, significant loss of visual field and central vision. Once such factors are identified, more appropriate screening and treatment recommendations can be made so that resources and treatments are appropriately allocated.

DAVID S FRIEDMAN

Wilmer Eye Institute, Johns Hopkins University, Baltimore, MD 21287, USA

1 Foster PJ, Oen FT, Machin D, et al. The prevalence of glaucoma in Chinese residents of Singapore: a cross-sectional population survey of the Tanjong residents of Singapore: a cross-sectional population

2 Dandona L, Dandona R, Mandal P, et al. Angle-closure glaucoma in an urban population in southern India. The Andhra Pradesh eye disease study. Ophthalmology 2000;107:1710-6.

3 Congdon NG, Quigley HA, Hung PT, et al. Screening techniques for angleclosure glaucoma in rural Taiwan. Acta Ophthalmol Scand 1996;74:113-19.

4 Alsbirk PH. Anatomical risk factors in primary angle-closure glaucoma. A ten year follow up survey based on limbal and axial anterior chamber depths in a high risk population. Int Ophthalmol 1992;16:265-72.

5 Foster PJ, Baasanhu J, Alsbirk PH, et al. Glaucoma in Mongolia. A population-based survey in Hovsgol province, northern Mongolia [see comments]. Arch Ophthalmol 1996;114:1235-41.

6 Lowe RF. Primary angle-closure glaucoma. Prevention and early treatment. Isr 7 Med Sci 1972;8:1362-5.

7 Edwards RS. Behaviour of the fellow eye in acute angle-closure glaucoma. Br F Ophthalmol 1982;6:576-9.

8 Snow JT. Value of prophylactic peripheral iridectomy on the second eye in angle-closure glaucoma. Trans Ophthalmol Soc UK 1977;97:189-91.

9 Fleck BW, Wright E, Fairley EA. A randomised prospective comparison of operative peripheral iridectomy and Nd:YAG laser iridotomy treatment of acute angle closure glaucoma: 3 year visual acuity and intraocular pressure control outcome. Br 7 Ophthalmol 1997;81:884-8.

10 Ang LP, Aung T, Chew PT. Acute primary angle closure in an Asian population: long-term outcome of the fellow eye after prophylactic laser population: long-term outcome of the fellow eye after

11 Wilensky JT, Kaufman PL, Frohlichstein D, et al. Follow-up of angle-closure glaucoma suspects. Am f Ophthalmol 1993;115:338-46. 
12 Alsbirk PH. Anatomical risk factors in primary angle-closure glaucoma. A ten year follow up survey based on limbal and axial anterior chamber depths in a high risk population. Int Ophthalmol 1992;16:265-72. primary angle closure in east Asian eyes. Br F Ophthalmol 2000;84:1255-9.

14 Alsagoff Z, Aung T, Ang LP, et al. Long-term clinical course of primary angle-closure glaucoma in an Asian population [In Process Citation]. Ophthalmology 2000;107:2300-4.

15 Caronia RM, Liebmann JM, Stegman Z, et al. Increase in iris-lens contact after laser iridotomy for pupillary block angle closure. Am $\mathcal{F}$ Ophthalmo 1996;122:53-7.

16 Robin AL, Pollack IP. A comparison of neodymium: YAG and argon laser iridotomies. Ophthalmology 1984;91:1011-16.
17 Quigley HA. Long-term follow-up of laser iridotomy. Ophthalmology 1981;88:218-24.

18 Jampel HD, Friedman DS, Lubomski LL, et al. Treatment of coexisting cataract and glaucoma. Agency for Healthcare Research Quality Contract No 290-097-0006.

19 Fleck BW, Wright E, McGlynn C. Argon laser pretreatment 4 to 6 weeks before Nd:YAG laser iridotomy. Ophthalmic Surg 1991;22:644-9.

20 Pollack IP. Laser iridotomy in the treatment of angle-closure glaucoma. Ann Ophthalmol 1981;13:549-50.

21 Schwartz GF, Steinmann WC, Spaeth GL, et al. Surgical and medical management of patients with narrow anterior chamber angles: comparative results. Ophthalmic Surg 1992;23:108-12.

\section{Video Reports (www.bjophthalmol.com)}

- Capsule staining and mature cataracts: a comparison of indocyanine green and trypan blue dyes. D F Chang

- Pearls for implanting the Staar toric IOL. D F Chang

- An intraocular steroid delivery system for cataract surgery. D F Chang

- Evaluation of leucocyte dynamics in mouse retinal circulation with scanning laser ophthalmoscopy. Heping Xu, A Manivannan, Garry Daniels, Fanet Liversidge, Peter F Sharp, fohn V Forrester, Isabel $\mathcal{F}$ Crane 